\title{
Quark Fragmentation to Pions in an Effective Chiral Theory
}

\author{
W. Bentz ${ }^{1, a}$, T. Ito ${ }^{1}$, I.C. Cloët ${ }^{2}$, A.W. Thomas ${ }^{3}$, and K. Yazaki ${ }^{4}$ \\ 1 Department of Physics, Tokai University, Hiratsuka-shi, Kanagawa 259-1292, Japan \\ 2 Department of Physics, University of Washington, Seattle, WA 98195-1560, USA \\ 3 School of Chemistry and Physics, University of Adelaide, Adelaide, SA 5005, Australia \\ 4 Radiation Laboratory, Nishina Accelerator Research Center, RIKEN, Wako, Saitama 351-0198, Japan
}

\begin{abstract}
A description of fragmentation functions which satisfy the momentum and isospin sum rules is presented in an effective chiral quark theory of QCD. We concentrate on the pion fragmentation function, taking into account cascade-like processes in a generalized jet-model approach. Numerical results obtained in this NJLjet model are presented and compared to empirical parametrizations.
\end{abstract}

\section{Introduction}

Quark distribution and fragmentation functions are the basic nonperturbative ingredients for a QCD-based analysis of hard scattering processes [1-3]. Distribution functions can be extracted by analyzing inclusive processes, and their description in terms of effective quark theories of QCD has been quite successful [4]. In recent years there has been a significant effort to extract the fragmentation functions by analyzing inclusive hadron production (semiinclusive) processes in $e^{+} e^{-}$annihilation, deep-inelastic lepton-nucleon scattering and proton-proton collisions [5, $6]$. Because of the importance of the fragmentation functions many attempts have been made to describe them using effective quark theories [7]. However, in these earlier attempts only the elementary fragmentation process $q \rightarrow$ $q \pi$ was considered, and the result did not satisfy the momentum and isospin sum rules in a natural way. In a recent publication [8] we have shown that cascade-type fragmentation chains must be included in order to satisfy these sum rules.

The purpose of this paper is to apply the method of the quark jet-model, as formulated originally by Field and Feynman [9], to calculate the spin-independent fragmentation functions to pions in the Nambu-Jona-Lasinio (NJL) model [10], which has proven to be a successful effective theory of QCD. We will introduce a generalized product ansatz to describe multi-fragmentation processes, and we will demonstrate that this NJL-jet model provides a very reasonable framework for describing the fragmentation functions.

\footnotetext{
a e-mail: bentz@keyaki.cc.u-tokai.ac.jp
}

\section{Operator definitions and sum rules}

The spin-independent fragmentation function for $q \rightarrow h$ is defined by

$$
\begin{aligned}
D_{q}^{h}(z) & =\frac{z}{12} \int \frac{d \omega^{-}}{2 \pi} e^{i p_{-} \omega^{-} / z} \hat{\sum}_{n} \\
& \times\left\langle p(h), p_{n}|\bar{\psi}(0)| 0\right\rangle \gamma^{+}\left\langle 0\left|\psi\left(\omega^{-}\right)\right| p(h), p_{n}\right\rangle .
\end{aligned}
$$

The field operators refer to a quark of flavour $q$, the symbol $p(h)$ refers to a hadron $h$ with momentum $p$, and $p_{n}$ labels the spectator state. If we introduce the Fourier decomposition of the "good" light-cone quark field operator, we obtain the expression

$$
D_{q}^{h}(z) d z=\frac{1}{6} d p_{-} \int d^{2} p_{\perp} \sum_{\alpha} \frac{\left\langle k(\alpha)\left|a_{h}^{\dagger}(p) a_{h}(p)\right| k(\alpha)\right\rangle}{\langle k(\alpha) \mid k(\alpha)\rangle} .
$$

Here $d z=d p_{-} / k_{-}$, implying $p_{-}=z k_{-}$for some fixed $k_{-}>0$. The creation and annihilation operators refer to the hadron $h$, and $k(\alpha)$ labels a quark state of flavour $q$ with momentum $k$ and spin-color $\alpha$. The result (2) can be interpreted as the light-cone momentum distribution of the hadron $h$ in the quark $q$. This interpretation provides a natural link to models describing the hadron cloud around constituent quarks, like the familiar pion cloud model.

The momentum and isospin sum rules obtained from Eq. (2) are

$$
\begin{aligned}
& \sum_{h} \int_{0}^{1} d z z D_{q}^{h}(z)=1, \\
& \sum_{h} \int_{0}^{1} d z t_{h} D_{q}^{h}(z)=t_{q} .
\end{aligned}
$$

The condition which lies at the basis of these sum rules is that the initial quark state is an eigenstate of the momentum and isospin operators, expressed solely in terms 
EPJ Web of Conferences

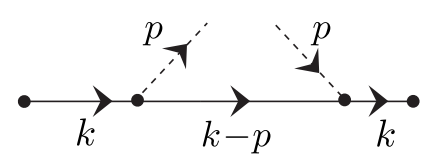

Fig. 1. Cut diagram for the elementary fragmentation function $d_{q}^{\pi}(z)$. Here $p_{-}=z k_{-}$and the two quark lines with momentum $k$ are connected by a $\gamma^{+}$.

of hadrons. The physical content of these sum rules is that $100 \%$ of the initial quark light-cone momentum $\left(k_{-}\right)$and isospin $\left(t_{q}\right)$ are transferred to the hadrons. (Note that the definition in Eq. (1) implies an average over the isospin of the soft quark remainder of a fragmentation chain.)

\section{Elementary fragmentation function}

The elementary fragmentation function for the pion is represented in Fig. 1 as a cut diagram, and is expressed by

$$
\begin{aligned}
d_{q}^{\pi}(z) & =\frac{1}{2}\left(1+\tau_{\pi} \tau_{q}\right) z g_{\pi}^{2} \int \frac{d^{2} \mathbf{p}_{\perp}}{(2 \pi)^{3}} \\
& \times \frac{\mathbf{p}_{\perp}^{2}+M^{2} z^{2}}{\left[\mathbf{p}_{\perp}^{2}+M^{2} z^{2}+(1-z) m_{\pi}^{2}\right]^{2}} .
\end{aligned}
$$

Here $M$ is the constituent quark mass, $g_{\pi}$ is the pion-quark coupling constant defined via the residue of the $q \bar{q} t$-matrix at the pion pole, and $\left(\tau_{u}, \tau_{d}\right)=(1,-1),\left(\tau_{\pi^{+}}, \tau_{\pi^{0}}, \tau_{\pi^{-}}\right)=$ $(1,0,-1)$. This elementary fragmentation function satisfies the following relation:

$$
\begin{aligned}
\int_{0}^{1} d z d_{q}^{\pi}(z) & =\frac{1}{3}\left(1+\tau_{\pi} \tau_{q}\right)\left(1-Z_{Q}\right) \\
& \Longrightarrow \int_{0}^{1} d z \sum_{\tau_{\pi}} d_{q}^{\pi}(z)=1-Z_{Q}
\end{aligned}
$$

where $Z_{Q}$ is the residue of the quark propagator in the presence of the pion cloud. (The corresponding Feynman diagram for the quark self energy is obtained by connecting the pion lines in Fig. 1.)

Because $Z_{Q}$ is interpreted as the probability to find a bare constituent quark without the pion cloud, Eq. (6) indicates that the elementary fragmentation function is normalized to the number of pions per quark. This is expected from our discussions in relation to Eq.(2). Because typical values of $Z_{Q}$ in models based on constituent quarks are between 0.8 and 0.9 , we see from Eq. (6) that the momentum sum rule $\int_{0}^{1} d z z \sum_{\tau_{\pi}} d_{q}^{\pi}(z)$ will be much smaller than typical empirical values. For example, the NLO analysis of Ref. [5] found a pion momentum sum of $\simeq 0.74$. From this we can anticipate that the elementary fragmentation function $d_{q}^{\pi}$ will be very small compared to the empirical one (see Section 5).

Although a description of fragmentation functions using only the elementary fragmentation processes does not violate any conservation law, it is completely inadequate for the following reasons: Firstly, there is a large probability $\left(Z_{Q}\right)$ that the initial quark does not fragment. Secondly, if it does fragment the momentum fraction $1-Z_{Q}$

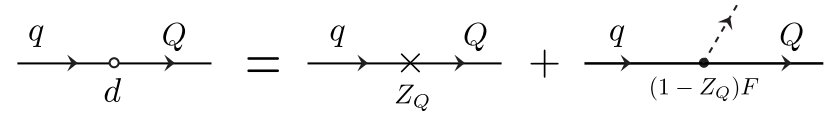

Fig. 2. Graphical representation of the two terms in the expression Eq.(7) for $d_{q}^{Q}(\eta)$.

is shared between the quark remainder and the pion. Both points are in contradiction to the usual assumption of complete hadronization, which is expressed by the momentum sum rule of Eq. (3).

\section{Generalized product ansatz for quark cascades}

From the previous section, it is clear that we have to consider the possibility that the fragmenting quark produces a cascade of mesons. Inspired by the quark jet-model of Field and Feynman [9], we will first introduce a generalized product ansatz to describe multifragmentation processes, and then explain its physical significance.

We first introduce an auxiliary quantity $d_{q}^{Q}(\eta)$, which describes the elementary fragmentation of a quark $q$ to another quark $Q$. (The variable $\eta$ is the light cone momentum fraction of $Q$ w.r.t. to the initial quark q.) This function, which is essentially the same as the distribution function of $Q$ inside $q$, is represented graphically by Fig.2, and expressed as follows ${ }^{1}$ :

$$
\begin{aligned}
6 d_{q}^{Q}(\eta) & =Z_{Q} \delta(\eta-1)+d_{q}^{\pi}(1-\eta) \\
& \equiv Z_{Q} \delta(\eta-1)+\left(1-Z_{Q}\right) F(\eta)
\end{aligned}
$$

The first term on the r.h.s. of Eq.(7), or Fig.2, refers to the process where the initial quark does not emit a pion. The second term, which refers to the pion emission process with probability $1-Z_{Q}$, is obtained by replacing $\eta \rightarrow$ $1-\eta$ in the elementary fragmentation function $d_{q}^{\pi}(\eta)$ of the previous section (apart from a similar replacement for isospin). By definition, the function $F(\eta)$ is normalized to 1.

Because the function $d_{q}^{Q}$ is the splitting function for the elementary process $q \rightarrow Q$, we make the following product ansatz for the total fragmentation function $D_{q}^{\pi}(z)$ :

$$
\begin{aligned}
D_{q}^{\pi}(z) & =\int_{0}^{1} \mathrm{~d} \eta_{1} \int_{0}^{1} \mathrm{~d} \eta_{2} \ldots \int_{0}^{1} \mathrm{~d} \eta_{N} \\
& \times 6 d\left(\eta_{1}\right) \cdot 6 d\left(\eta_{2}\right) \ldots 6 d\left(\eta_{N}\right)\left(\sum_{m=1}^{N} \delta\left(z-z_{m}\right)\right) .
\end{aligned}
$$

Here we introduced a parameter $N$, which is the maximum number of pions which can be produced by the initial quark. The product ansatz (8) is shown graphically in Fig.3.

1 For simplicity we do not write out the explicit isospin dependence on the r.h.s. of Eq.(7). The full expressions can be found in Ref.[8]. 


\section{$19^{\text {th }}$ International IUPAP Conference on Few-Body Problems in Physics}

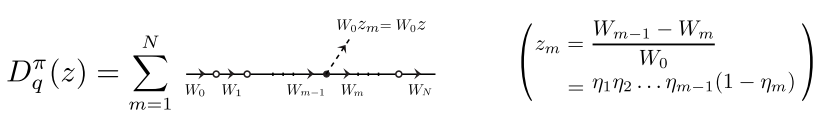

Fig. 3. Graphical representation of the product ansatz Eq.(8).

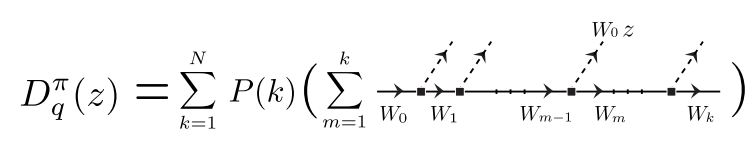

Fig. 4. Graphical representation of Eq.(9).

To see the physical meaning of this ansatz, we use Eq.(7) to rewrite Eq.(8) identically as follows:

$$
\begin{aligned}
D_{q}^{\pi}(z) & =\sum_{k=1}^{N} P(k) \int_{0}^{1} \mathrm{~d} \eta_{1} \int_{0}^{1} \mathrm{~d} \eta_{2} \ldots \int_{0}^{1} \mathrm{~d} \eta_{k} \\
& \times F\left(\eta_{1}\right) \cdot F\left(\eta_{2}\right) \ldots F\left(\eta_{k}\right)\left(\sum_{m=1}^{k} \delta\left(z-z_{m}\right)\right) .
\end{aligned}
$$

This expression is represented graphically by Fig. 4. The binomial probability distribution that $k$ pions are produced for a maximum of $N$ pions is given by

$$
P(k)=\left(\begin{array}{c}
N \\
k
\end{array}\right) Z_{Q}^{N-k}\left(1-Z_{Q}\right)^{k}
$$

and satisfies the normalization condition $\sum_{k=0}^{N} P(k)=1$. It is well known that in the limit $N \rightarrow \infty$ the binomial distribution (10) becomes a normalized Gauss (normal) distribution with the same mean number and variance as the original binomial distribution.

In order to see whether the momentum sum rule (3) is satisfied by the fragmentation function (9), we note that in each elementary fragmentation process, a fraction $\alpha \equiv$ $\langle z F(z)\rangle$ is left to the quark, where $\langle\ldots\rangle$ means an integration over $z$. Therefore the momentum fraction left to the final quark remainder after emission of a maximum of $N$ pions is given by

$$
1-\int_{0}^{1} \mathrm{~d} z \sum_{\tau_{\pi}} z D_{q}^{\pi}(z)=\sum_{k=0}^{N} P(k) \alpha^{k} .
$$

In order to satisfy the momentum sum rule (3), this should vanish. It is easy to see that (11) vanishes only in the limit $N \rightarrow \infty$, because in this limit $P(k)$ becomes a normal distribution with mean value $\langle k\rangle=N\left(1-Z_{Q}\right) \rightarrow \infty$, and then the functions $P(k)$ and $\alpha^{k}$ have zero overlap. Therefore, in the limit that the maximum number of mesons which can be produced by the fragmenting quark is assumed to be infinite, $100 \%$ of the initial quark momentum is transfered to the mesons, and the sum rule (3) is satisfied.

We note that in the limit $N \rightarrow \infty$, the fragmentation function (9) becomes essentially equivalent to the original infinite product ansatz of Field and Feynman [9], because in this limit $P(k)$ is effectively zero for finite $k$, i.e., the probability of the fragmenting quark to emit a finite number of mesons is zero.
In the actual calculation it is not necessary to evaluate the products (9) explicitly, because a compact integral equation for the fragmentation function can be derived. We refer to Ref.[8] for the explicit form of this integral equation, as well as for the verification of the isospin sum rule (4).

\section{Numerical results and discussions}

In this Section we present the numerical results for the fragmentation function in the NJL-jet model, which was developed in the previous section. We will use the same regularization scheme as in Ref. [11], namely the invariant mass, or Lepage-Brodsky (LB) [12] regularization scheme, with the same values of the constituent quark mass $(M=$ $300 \mathrm{MeV})$ and the equivalent 3-momentum cut-off $\left(\Lambda_{3}=\right.$ $670 \mathrm{MeV})$, which is determined by reproducing the experimental pion decay constant. The LB regularization scheme is suitable for regularizing integrals in terms of light cone variables, and preserves the sum rules. We did not investigate whether other parameter sets or other regularization schemes lead to a better description of the fragmentation functions.

As usual, we will associate a low energy renormalization scale $\left(Q_{0}^{2}\right)$ to our NJL results and evolve them in $Q^{2}$ by using the QCD evolution equations. We will use the value $Q_{0}^{2}=0.18 \mathrm{GeV}^{2}$, which was determined in Ref. [11] from a comparison with the empirical distribution functions at a high energy scale $Q^{2}=4 \mathrm{GeV}^{2}$. For the evolution of the fragmentation functions we limit ourselves to the leading order (LO) in $\alpha_{s}$. For this purpose, we use the $Q^{2}$ evolution code of Ref. [13] at LO for the distribution functions, and perform the transformation of the kernels as explained in Ref.[5] or Ref.[8]. (Unfortunately, a next-toleading (NLO) evolution code for the fragmentation functions is not yet publicly available. In this paper we do not attempt a quantitative comparison with the empirical functions, therefore we leave the NLO calculation to a future work.)

Fig. 5 shows the favoured fragmentation function $z D_{u}^{\pi^{+}}(z)$, and Fig. 6 shows the unfavoured fragmentation function $z D_{\bar{u}}^{\pi^{+}}(z)$. The elementary fragmentation function for the favoured case is very small, as is expected from our discussions in Sect. 3, while for the unfavoured case it vanishes identically because of charge conservation. The results shown in these figures show the tremendous enhancement of the fragmentation functions caused by the cascade-type processes. The final results shown by the solid lines have the correct order of magnitude for intermediate and large $z$, when compared with the empirical functions. This point, which reflects the fact that our model satisfies the momentum sum rule, is very important, because effective quark model calculations completed hitherto only considered the elementary fragmentation functions and introduced some ad hoc parameters (like normalization constants) to obtain the correct order of magnitude.

Quantitatively, Fig. 5 indicates that our favoured fragmentation function is too big at large $z$ and too small at smaller $z$. This is natural for the following reasons: Firstly, 


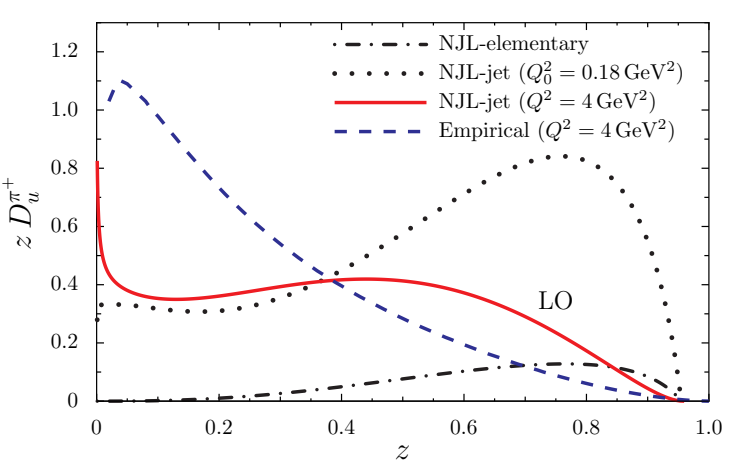

Fig. 5. Favoured fragmentation function $z D_{u}^{\pi^{+}}(z)$. The dashdotted line is the elementary fragmentation function, and the dotted line is the full fragmentation function in the NJL-jet model. The solid line is the result after $\mathrm{LO}$ evolution to $Q^{2}=4 \mathrm{GeV}^{2}$, and the dashed line is the empirical NLO result of Ref. [5], evolved to $Q^{2}=4 \mathrm{GeV}^{2}$.

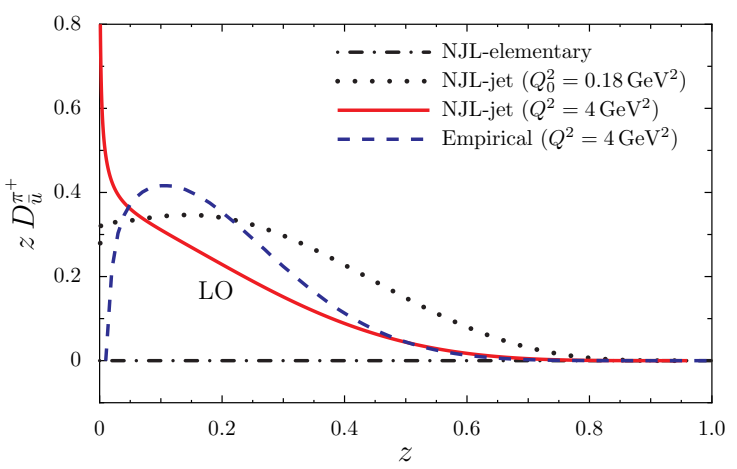

Fig. 6. Unfavoured fragmentation function $z D_{\bar{u}}^{\pi^{+}}(z)$. The dotted line is the result in the NJL-jet model. The solid line is the result after $\mathrm{LO}$ evolution to $Q^{2}=4 \mathrm{GeV}^{2}$, and the dashed line is the empirical NLO result of Ref. [5], evolved to $Q^{2}=4 \mathrm{GeV}^{2}$. Note that the elementary fragmentation function vanishes for this case because of charge conservation.

we can expect that a NLO calculation will lead to a softening of the fragmentation functions. Secondly, some of the observed pions are secondary ones, which come from the decay of primary $\rho$ and $\omega$ mesons. Thirdly, the coupling to other fragmentation channels, in particular the nucleon, antinucleon and kaon, will transfer some amount of the hard quark momentum to these other hadrons. Also, one should not forget that the empirical fragmentation functions have very large uncertainties, which are not indicated in our figures. Nevertheless, Figs. 5 and 6 indicate that the present NJL-jet model provides a reasonable starting point for the description of fragmentation functions.

\section{Summary and conclusions}

In this paper we used the NJL model as an effective quark theory to study the simplest fragmentation function, namely, the fragmentation of unpolarized quarks to pions. Our aim was to develop a framework which satisfies the momentum and isospin sum rules in a natural way, without the introduction of ad hoc parameters. This framework should also give fragmentation functions that have the correct order of magnitude at intermediate and large $z$. We explained in detail, that for this purpose, the simplest approximation where a truncation is made to the one-quark spectator state in the defining relation given by Eq.(1), is completely inadequate. Although this approximation does not violate any conservation law, it gives very small fragmentation functions; because the probability for the elementary fragmentation process is small in effective theories based on constituent quarks and the quark remainder can carry an appreciable amount of momentum.

In order to overcome these difficulties we followed the idea of the quark jet-model and made a generalized product ansatz to describe the cascade processes in the NJL model. We explained that this ansatz corresponds to a binomial distribution for the number of mesons emitted from the quark. However, in the limit that the maximum number of mesons becomes very large the results are independent of the form of this distribution function. Our formulation thus represents an extension of the original quark jet-model, which assumed an infinite number of mesons from the outset. We have shown that this NJL-jet model describes fragmentation processes where $100 \%$ of the initial quark light-cone momentum is transferred to mesons. The momentum sum rule of Eq.(3), which is assumed valid in all QCD based empirical fits, is then satisfied automatically without introducing any new parameters into the theory. We have also shown that the isospin sum rule of Eq.(4) is naturally satisfied in this approach.

The comparison with the empirical fragmentation functions shows that our calculated functions have the correct order of magnitude for intermediate and large $z$. We highlighted that a straightforward extension to include the NLO terms in the $Q^{2}$ evolution and to include the effect of primary $\rho$ and $\omega$ mesons, as well as fragmentation to other hadronic channels, will improve the description. Therefore we can conclude that our NJL-jet model provides a reasonable framework to analyse fragmentation functions in an effective quark theory.

\section{Acknowledgments}

This work was supported by the Grant in Aid for Scientific Research of the Japanese Ministry of Education, Culture, Sports, Science and Technology, Project No. C19540306 and by the U.S. Department of Energy Grant No. DEFG03-97ER4014, and by the Contract No. DEAC05-06OR23177, under which Jefferson Science Associates, LLC operates Jefferson Laboratory.

\section{References}

1. R. D. Field and R. P. Feynman, Phys. Rev. D 15, 2590 (1977).

2. J. C. Collins and D. E. Soper, Nucl. Phys. B 194, 445 (1982). 
$19^{\text {th }}$ International IUPAP Conference on Few-Body Problems in Physics

3. R. K. Ellis, W. J. Stirling and B. R. Webber, "QCD and collider physics", Cambridge University Press, 1996.

4. I. C. Cloët, W. Bentz and A. W. Thomas, Phys. Lett. B 621, 246 (2005).

5. M. Hirai, S. Kumano, T. H. Nagai and K. Sudoh, Phys. Rev. D 75, 094009 (2007).

6. D. de Florian, R. Sassot and M. Stratmann, Phys. Rev. D 75, 114010 (2007); S. Kretzer, E. Leader and E. Christova, Eur. Phys. J. C 22, 269 (2001).

7. R. Jakob, P. J. Mulders and J. Rodrigues, Nucl. Phys. A 626, 937 (1997); D. Amrath, A. Bacchetta and A. Metz, Phys. Rev. D 71, 114018 (2005); A. Bacchetta, L. P. Gamberg, G. R. Goldstein and A. Mukherjee, Phys. Lett. B 659, 234 (2008).

8. T. Ito, W. Bentz, I.C. Cloët, A.W. Thomas, K. Yazaki, Phys. Rev. D 80, 074008 (2009).

9. R. D. Field and R. P. Feynman, Nucl. Phys. B 136, 1 (1978).

10. Y. Nambu and G. Jona-Lasinio, Phys. Rev. 122, 345 (1961); Phys. Rev. 124, 246 (1961).

11. W. Bentz, T. Hama, T. Matsuki and K. Yazaki, Nucl. Phys. A 651, 143 (1999).

12. G.P. Lepage and S.J. Brodsky, Phys. Rev. D 22, 2157 (1980).

13. M. Miyama and S. Kumano, Comput. Phys. Commun. 94, 185 (1996). 